\title{
FIELD TEST OF 4G LTE 1800 MHz DEPLOYMENT IN DENSE-URBAN ENVIRONMENT IN THAILAND
}

\author{
Settapong Malisuwan ${ }^{1}$, Dithdanai Milindavanij ${ }^{2}$, Noppadol Tiamnara ${ }^{3}$ \\ ${ }^{1,2,3}$ The National Broadcasting and Telecommunications Commission (NBTC), Thailand
}

\begin{abstract}
LTE as a high speed and high bandwidth technology is not expected to supersede $3 G$ but rather to complement it, as consumers' demand for data is insatiable. Therefore, operators globally are rushing for LTE deployment in order to increase data supply both in terms of bandwidth and speed. In this paper, the National Broadcasting and Telecommunications Commission of Thailand provides the results of LTE deployment field test in order to educate the academia and stakeholders in the telecommunications industry on LTE deployment standards and to contribute to further research on LTE deployment. An end to end LTE system was setup using a $2 \times 15 \mathrm{MHz}$ bandwidth in the $1800 \mathrm{MHz}$ band, and measurement were taken to assess system performance and the applicability of LTE system operations. The results of the field test shows that the LTE system has sufficient performance for use in the field. The drive test performed was with only one UE active in the cell. Testing of applications traditional used in a fixed internet protocol network showed that it could provide excellent results even on the LTE system.
\end{abstract}

Key Words: Field Test, LTE, Dense - urban, Throughput

\section{INTRODUCTION}

LONG term Evolution (LTE) has expeditiously penetrated the market at a much faster rate than UMTS did about a decade ago. Initially, operators invested in UMTS presuming its success, but demand unexpectedly remained low. Globally, all operators have acknowledged that LTE is critical to satisfy the already rapidly growing and unprecedented demand for mobile data [1].

Though 3GPP standard specified several spectrum bands that are recommended for LTE deployment, $2600 \mathrm{MHz}$ and $1800 \mathrm{MHz}$ seems to be the most apt band due to its distinctive properties [3]. Apart from $2600 \mathrm{MHz}, 1800 \mathrm{MHz}$ is also highly appropriate since it is capable of providing better outdoor and indoor coverage propagation parameters [2].

Operators all around the world are accelerating LTE deployments. LTE deployments are usually implemented on 10 or $20 \mathrm{MHz}$ of free spectrum on $1800 \mathrm{MHz}$. Majority of European countries have encouraged operators to start deploying LTE and permitted the coexistence between GSM and LTE on $1800 \mathrm{MHz}$ spectrum band. To emphasize, regulators are playing a major role in fostering the adoption and deployment of 4G LTE networks by making network capacity solutions available. Therefore, ambiguity in regulations and complexity in fragmentation of LTE frequencies will continue to impede the development of LTE-compatible consumer devices.

In Thailand, the Act on Organization to Assign Radio Frequency and to Regulate the Broadcasting and Telecommunications Services B.E. 2553 (2010) was established, which stipulates that spectrum licenses must be issued via auctions only. The National Broadcasting and
Telecommunications Commission (NBTC) has duties, by the law, in spectrum management. The objectives of spectrum assignment must be conformed with policy objectives stipulated in relevant laws. The NBTC held the country's first spectrum auction, for the $2.1 \mathrm{GHz}$ spectrum, in 2012. Around the same time, the different BTO contracts between the private operators and state enterprises began to expire starting in 2013. By law these spectrum must be returned to the NBTC to be re-allocated through the auction process. After the BTO expiration, NBTC assigned the $4 \mathrm{G}$ $1800 \mathrm{MHz}$ spectrum to 2 licensees by the spectrum auction process. The license details is presented in table 1 .

Table 1: The Licensees and bands of the $1800 \mathrm{MHz}$ spectrum

\begin{tabular}{|c|c|}
\hline Spectrum Lot & Licensee \\
\hline Lot $1: 1710-1725 \mathrm{MHz} /$ & TrueMove H Universal \\
$1805-1820 \mathrm{MHz}$ & Communications \\
\hline Lot $2: 1725-1740 \mathrm{MHz} /$ & Advance Wireless \\
$1820-1835 \mathrm{MHz}$ & Network \\
\hline
\end{tabular}

The National Broadcasting and Telecommunications Commission has cooperated with the operators jointly launched high speed wireless broadband field test using Long Term Evolution (LTE) technology in downtown area of Bangkok. This field test utilized $1800 \mathrm{MHz}$ frequency band to conduct the LTE test and this study seeks to present the engineering properties of transmitting and receiving frequency signals of LTE technology and reception capability of signals in various distances.

Besides, this field test is conducted to help conclude and develop a guideline for the design and establishment of network infrastructure and quality of service in LTE. The 
LTE field test on $1800 \mathrm{MHz}$ with $2 \times 15 \mathrm{MHz}$ bandwidth is presented in this study in detail.

\section{SYSTEM CHARACTERISTIC AND TEST PROCEDURE}

The 4G LTE field test took place in Bangkok downtown area as shown in Fig 1. The test is categorized into two phases, first is the coverage test which is followed by the traffic test. The coverage test examined the $1800 \mathrm{MHz}$ LTE service area for signal strength in the public area and indoor area. The traffic test assessed the usage properties of LTE technology in the following aspects.

- High data rates up to $150 \mathrm{Mbps} \mathrm{DL}$ and $50 \mathrm{Mbps} \mathrm{UL}$ such as dual downlink antenna, 64-QAM Downlink and 16- QAM Uplink.

- Intra LTE mobility for LTE network handover testing.

- Self Organizing Network (SON) test for LTE network management testing.

- Interoperability test for the ability of current network and LTE network to work harmoniously together.

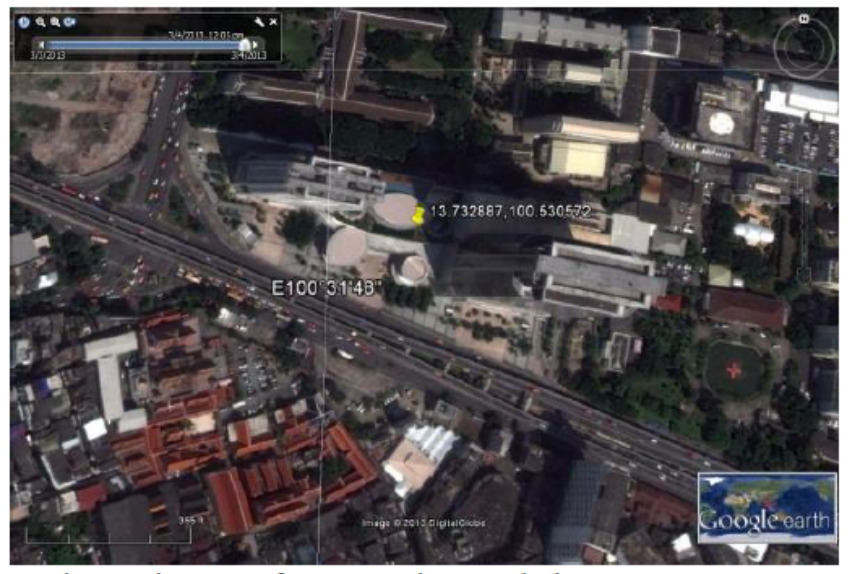

Fig. 2 Diagram of LTE test in Bangkok Downtown Area

Information on LTE Field Test is summarized in Table 1 below.

Table 1. Information on LTE Field Test

\begin{tabular}{|c|c|c|c|}
\hline \multirow{4}{*}{ Frequency } & Bandwidth & \multicolumn{2}{|c|}{$2 \times 15 \mathrm{MHz}$} \\
\cline { 2 - 4 } & $\begin{array}{c}\text { Technology } \\
\text { Used }\end{array}$ & \multicolumn{2}{|c|}{ FDD } \\
\hline \multirow{4}{*}{ Equipment } & \multirow{4}{*}{ Base Station } & Brand/Model & $\begin{array}{c}\text { Ericsson } \\
\text { RBS6201/RBS6601 }\end{array}$ \\
\cline { 3 - 4 } & & TX Power & $20+20 \mathrm{~W}$ \\
\cline { 3 - 4 } & & Antenna Height & $29-43 \mathrm{~m}$ \\
\cline { 3 - 4 } & & Antenna Gain & $9-17.7 \mathrm{dBi}$ \\
\cline { 3 - 4 } & & Antenna Beam & $65-68 \mathrm{deg}$ \\
\cline { 3 - 4 } & \multirow{2}{*}{ Terminal } & Brand/Model & Huawei E392u-12 \\
\cline { 3 - 4 } & & LTE Terminal Type & USB \\
\hline
\end{tabular}

For the LTE field test, the equipment chosen by MNO is from Ericsson and setup 5 eNodeB base stations were utilized. At switching office, the core network (EPC: Evolved Packet Core) comprised of Serving Gateway (S-
GW), Packet Gateway (P-GW) and Mobility Management Entity (MME), together with Home Subscriber Server (HSS) and Operation Support Systems (OSS), were setup to link with the base stations via IP transmission (IP RAN) as illustrated in Fig 2.

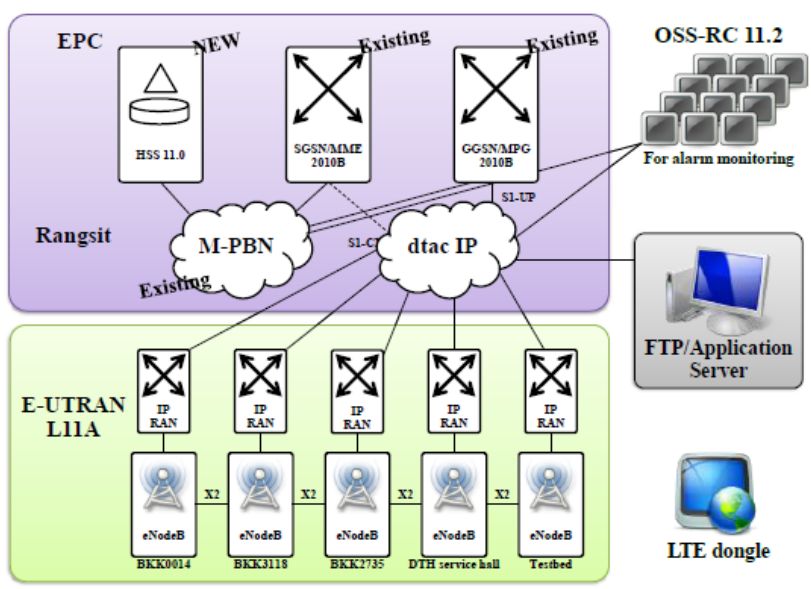

Fig. 3 LTE Network Test

Diagram showing LTE Network Under Test

\section{RESULTS AND DISCUSSION}

\section{A. Drive Test}

The drive test was on public roads along Rama IV road, between 10 to 700 meters away from the BTS eNodeB. This area is characterized by high building density along the road. This is a typical dense-urban deployment condition. The measurement scenario was performed on the route with only one UE (single user) active in the cell. The purpose of the single user test was to measure the achievable DL throughput rate in a typical urban cell with a cell radius of approximately 700 meters under highly varying channel conditions and typical urban speeds of up to $40 \mathrm{~km} / \mathrm{h}$.

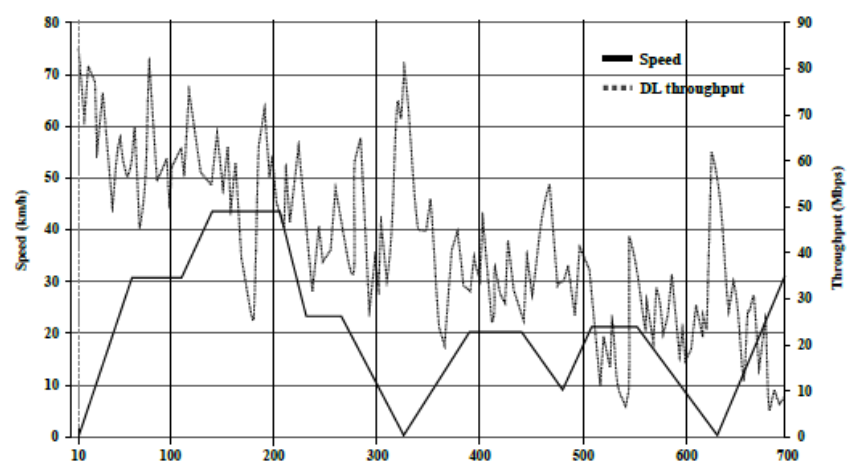

Fig. 4 Graph Displaying LTE Deployment DL Throughput

Fig. 4 illustrates the received DL throughput along the route together with the speed of the measurement van at the related spots. Since this route encompasses the public area and streets, there were several stops during traffic jams and traffic lights. As the measurement was performed at a time with high traffic density the average speed was only 20-40 $\mathrm{km} / \mathrm{h}$. The measured DL throughput was in the range of 8-85 Mbps. It varied extensively depending the shadowing 
conditions along the test route and decreased on average with the increasing distance from the site. Even at a distance of about 700 meters from the BTS eNodeB the average DL throughput reaches a level of $10 \mathrm{Mbps}$.

The result of the Long Term Evolution (LTE) technology trial is explained in detail.

1) Maximum receiving data rate (Downlink) was 99.8 Mbps at the application layer for CAT3 Aircard.

2) Maximum transmitting data rate (Uplink) was $49.4 \mathrm{Mbps}$ at the application layer for CAT3 Aircard.

3) Continuous receiving and transmitting data in multiple simultaneous sessions were able to last over 10 minutes.

4) Maximum data receiving and transmitting speed was achieved continuously.

5) Handover 0209 of transmit and received data rate between stations and within stations did not affect the user's attention (Hand Over Interruption Time as low as $50 \mathrm{~ms}$.)

6) Internet access speed was very fast at the level of $28 \mathrm{~ms}$. (Ping Time to Internet and Internal Server)

7) Drive and walk test results were closely similar to those of the model from 3GPP (EVA70 and EPA5)

8) In Multi User Test, six simultaneous terminals in various radio scenarios showed aggregate downlink throughput of $69.3 \mathrm{Mbps}$ and aggregate uplink throughput of 32.8 Mbps.

The interference analysis showed that there was no interference in the areas of drive and walk test for LTE on the $1800 \mathrm{MHz}$ frequency band. The test meets the required standard since the project utilized the LTE technology which complies with the 3GPP standard, therefore, the trial is considered to be reliable and is a success. Moreover, in terms of the equipment availability analysis, currently there are many equipment that are capable of utilizing LTE technology and those equipment are also able to operate in $2 \mathrm{G}$ and $3 \mathrm{G}$ network. Thus, this would allow users to use the equipment overseas while still benefiting from uninterrupted data access. At present, there are many manufacturers that produce LTE technology equipped devices. Consequently, this gives MNO variety of choices in choosing equipment to operate in their networks. In regards to the mobility analysis, this study also tested for mobility and handover. Illustrated in Fig 2, is the mobility test for LTE Trial. TEMS investigation 13.1 was used for testing the Test Bed and for mobility drive tests. It involves 1 laptop and $1 \mathrm{UE}$.

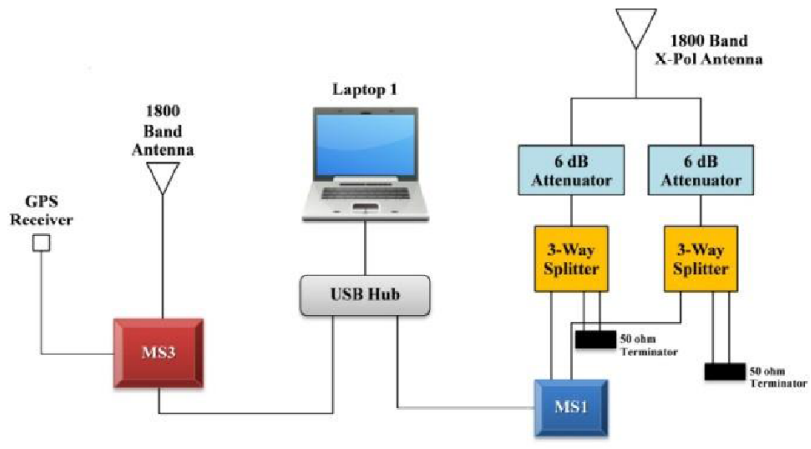

Fig. 4 Mobility test for LTE Trial
The test for handover was conducted in two ways. First is the handover between two cells within the same station and second is the handover between two cells from different stations. The handover test achieved successful results as $100 \%$ as the handover interruption time was low in the level that would never caught attention of users for both of the scenarios.

The results also indicated that performance of data reception and transmissions were just below $100 \mathrm{Mbps}$ and $50 \mathrm{Mbps}$, respectively. In addition, the outcome of the eNodeB performance by measuring various Key Performance Indicators (KPIs) according to the calculation of LTE is shown in a table 2.

\begin{tabular}{lc}
\multicolumn{2}{c}{ TABLE 2 } \\
KPI \\
\hline \hline & General \\
& Performance \\
& KPI \\
\hline System Availability (\%) & 100 \\
Accessibility (\%) & 100 \\
Mobility (\%) & - \\
Minute Per Abnormal Release (MPAR) & 49.06 \\
Retainability (\%) & 98.82 \\
\hline \hline
\end{tabular}

As shown in Table 2, system availability is defined as the percentage of time, within a given measurement period, that a cell or measurement area is available to accept traffic. Accessibility measured the successful rate attempted to communicate between equipment and stations. Moreover, MPAR is the ratio of the total end user time where a data session is maintained after being successfully set up, divided by the abnormal call disconnections. It represents the average length of time a data session call can be held by the end user before it is abnormally terminated. As for retainability, this is a value showing ability to communicate with the station.

Furthermore, capacity analysis is an important part that the high speed wireless broadband service providers must pay attention to as there must be a network service plan. In other words, the service providers must estimate numbers of devices that the system can handle, which depends on many factors and details that must be taken into consideration. The following are the factors to consider.

- System configuration information such as system bandwidth and modulation scheme, etc.

- Types/service categories such as Broadband Web Access (Web Browsing, FTP Upload/Download, etc.), Voice over IP and video streaming, etc.

- Guaranteed QoS values to the devices

- Physical status of devices such as distance between devices and stations, types of device, indoor/outdoor (LOS/NLOS) and mobility of devices, etc.

- Behavior of users such as frequent and duration of usage, etc.

From the above factors, the service providers must assess these prior to the design of the network. Moreover, this cannot be calculated or designate a value. 
Apart from above analysis, an application analysis was also part of the test as this is vital that various applications must be able run smoothly with least interruption. The result of this analysis was in satisfactory level.

\section{CONCLUSIONS}

Consumer behavior has transitioned from voice-centric service to multimedia or heavy data consuming services, thus the need for LTE deployment. In essence, LTE is critical to cope with the already existing rapid growth in demand for mobile data. The $1800 \mathrm{MHz}$ is one of the most preferential bands for LTE of its capability in catering to high bandwidth consuming or advanced technologies because of wide bandwidth of $2 \times 75 \mathrm{MHz}$. Therefore, 1800 $\mathrm{MHz}$ is widely used for LTE deployment all over the world.

This paper describes some of the field-performance DL throughput measurements of LTE field test in the denseurban environment in Bangkok while traveling at velocities of up to $50 \mathrm{~km} / \mathrm{h}$ and at distances of up to $700 \mathrm{~m}$. This research also provides the outcome of the eNodeB performance by measuring various Key Performance Indicators (KPIs) such as the accessibility rate, retainability rate, and the successful rate of handover. This paper will be beneficial to operators and regulators to prepare Thailand entering to ubiquitous society. In summary, the results show that the design goals of LTE can be met with a great deal of certainty and reliability. The LTE system is essential to provide adequate support for the high data rates required.

LTE as a technology is widely discussed within the literature; however, there is little academic research that discusses the real-world performance of LTE. The results of LTE performance provided within this research should be a good basis for engineers and operators who seek to repeat the test and it also contributes as a basis for further innovations. This research also contributes to the knowledge of regulators on LTE deployment standards.

\section{REFERENCES}

[1]. H.Kiyangagi and M. Minowa, "Field Trial for LTE Mobile Network System," FUJITSU Sci. Tech.J., vol 48, no.1, pp. 76-82, January 2012

[2]. P.M. Sainju, "LTE performance analysis on 800 and $1800 \mathrm{MHz}$ bands, Master of Science Thesis, Faculty Council of Computer and Electrical Engineering, Tampere University of Technology, Finland, September 2012

[3]. S. Sesia, I. Toufik, and M. Baker, LTE, The UMTS Long Term Evolution: From Theory to Practice. John Wiley \& Sons, 2009 